\title{
FIXAÇÃO DE FÓSFORO POR UM LATOSSOLO DO ESTADO DE MINAS GERAIS*
}

\author{
AIRTON MANSANO ** \\ HeLVÉCIO DE POLLI ** \\ Jesiel C. Freire** \\ LAFAYETTE F. SOBRAL ** \\ MAURÍCIO DE SOUZA ** \\ NELSON VENTORIN ** \\ Francisco DE A. F. DE Mello**
}

\section{RESUMO}

Foi estudada a adsorção do fósforo por amostras de três horizontes, $A_{1}(0-22 \mathrm{~cm}), A_{3}(22-56 \mathrm{~cm})$ e $B_{22}(155-200 \mathrm{~cm})$, de um Latossolo do Estado de Minas Gerais por meio da isoterma de Langmuir.

Os valores de adsorção máxima (b) e da constante de seletividade ( $\mathrm{K}$ ) calculados a partir da forma linear da equação de Langmuir foram correlacionados com algumas característicar físicas e químicas apresentadas pelos citados horizontes.

Os resultados encontrados permitiram concluir que:

a - As isotermas de adsorção mostraram duas regiões distintas: aquela em que o fosfato é fortemente retido foi convenientemente descrita pela equação de Langmuir.

b - Em virtude da diversidade das características físicas e químicas dos horizontes houve grande variação nos valores de adsorção máxima (b) e da constante de seletividade $(K)$.

\section{INTRODUÇÃO}

A adsorção do fosfato pelas partículas do solo é um fenômeno há muito conhecido. Entretanto, apesar do grande número de trabalhos

* Entregue para publicação em 30.12.1976.

* Alunos do Curso de Pós-Graduação em Solos e Nutrição de Plantas da Escola Superior de Agricultura "Luiz de Queiroz".

** Professor da disciplina Fertilidade do Solo do Curso referido. 
já executados para esclarecer esse assunto da físico-química do solo, que tem elevada importância sobre a fertilidade do mesmo, muito pouco se conhece ainda, acerca de seus detalhes.

Neste trabalho se pretendeu alcançar dois objetivos:

a - determinar a adsorção máxima e as constantes de seletividade de três horizontes de um Latossolo do Estado de Minas Gerais, através da isoterma de Langmuir.

b - correlacionar os valores encontrados com algumas características físicas e químicas dos mesmos horizontes.

Como já foi referido, muitos estudos já foram efetuados para se obter um melhor conhecimento sobre a adsorção do fosfato pelo solo. Ultimamente, a isoterma de Langmuir tem sido muito utilizada com essa finalidade. No Brasil, entre os poucos autores que se valeram desse processo podem ser citados SÁ JR. et al (1968), LOURENÇO (1973), BITTENCOURT \& ZAMBELLO (1973) e LEAL \& VELOSO (1973).

\section{MATERIAIS E MÉTODOS}

Foram utilizadas amostras dos horizontes $A_{1}(0-22 \mathrm{~cm}), A_{3}(22-56 \mathrm{~cm})$ e $B_{22}(155-200 \mathrm{~cm})$ de um Latossolo Roxo Distrófico do Município de Laras, Estado de Minas Gerais, cujas características referidas neste trabalho estão na Tabela 1.

Tabela 1 - Características dos horizontes $A_{1}, A_{3}$ e $B_{22}$ utilizados no trabalho.

\begin{tabular}{lccc}
\hline & & \multicolumn{2}{c}{ Horizonte } \\
\cline { 2 - 4 } Características & $\mathrm{A}_{1}$ & $\mathrm{~A}_{3}$ & $\mathrm{~B}_{22}$ \\
\hline \hline $\mathrm{Argila} \%$ & 82,65 & 87,15 & 86,05 \\
$\mathrm{St}, \mathrm{m}^{2} / \mathrm{g}$ & 53,00 & 76,00 & 63,00 \\
$\mathrm{pH}$ & 4,6 & 4,8 & 5,0 \\
$\mathrm{C} \%$ & 3,39 & 1,88 & 0,85 \\
$\mathrm{Fe} \mathrm{O}_{3}$ livre & 22,15 & 24,55 & 24,15 \\
$\mathrm{CTC}$ & 16,00 & 10,60 & 6,60 \\
$\mathrm{Ki}$ & 1,57 & 1,09 & 1,02 \\
$\mathrm{Kr}$ & 1,02 & 0,70 & 0,67 \\
\hline
\end{tabular}

Os métodos empregados para a obtenção dos dados da Tabela 1 são descritos a seguir. 
Argila \% - Método da pipeta (GROHMANN \& VAN RAIJ, 1974).

St, $\mathrm{m}^{2} / \mathrm{g}$ - Método proposto por HEILMAN et al (1965) que utiliza como fase adsorvida o éter monoetílico do etileno glicol (2-etoxietanol) (EMEG); o cálculo da superfície específica foi feito pela fórmula enpregada por GROHMANN (1972).

$$
\text { St, } \mathrm{m}^{2} / \mathrm{g}=\frac{\mathrm{mg} \text { EMEG } / \mathrm{g} \text { de terra }}{2,86 \times 10^{-4} \mathrm{~g} / \mathrm{m}^{2}}
$$

pH - Relação terra - água igual a 1:2,5 (VETTORI, 1969).

C \% - Combustão úmida com ácido crômico (VETTORI, 1969).

$\mathrm{Fe}_{2} \mathrm{O}_{3}$ livre - Redução do $\mathrm{Fe}$ com ditionito de sódio e complexão com citrato de sódio $0,3 \mathrm{M}$ e bicarbonato de sódio $1 \mathrm{M}$ (JACKSON, 1965). O desenvolvimento da cor foi obtido com o emprego de ácido ascórbico, 1-10 fenantrolina a $0,25 \%$ e citrato de sódio a $25 \%$, fazendo-se a leitura em fotocolorímetro.

CTC - Extração com acetato de amônio a pH 7,0 (VETTORI, 1969).

$\mathrm{Ki}$ e $\mathrm{Kr}$ - Ataque da amostra de T.F.S.A com $\mathrm{H}_{2} \mathrm{SO}_{4} \mathrm{~d}=1,47$, a quente sob refluxo. No extrato determinaram-se teores de $\mathrm{SiO}_{2}, \mathrm{Al}_{2} \mathrm{O}_{3}$ e $\mathrm{Fe}_{2} \mathrm{O}_{3}$ (VETTORI, 1969).

Para a determinação da adsorção máxima foi utilizada a metodologia proposta por OLSEN \& WATANABE (1957) que faz uso da equação de Langmuir a qual, na sua forma linear, é dada pela seguinte expressão:

$$
\frac{\mathrm{C}}{\mathrm{x} / \mathrm{M}}=\frac{1}{\mathrm{~Kb}}+\frac{1}{\mathrm{~b}} \mathrm{C}
$$

$\mathbf{C}=$ concentração de $\mathrm{P}$ na solução de equilíbrio.

$\mathrm{x} / \mathrm{M}=$ quantidade de $\mathrm{P}$ adsorvido por unidade de peso de solo (ug $\mathrm{P} / \mathrm{g}$ de solo).

$\mathrm{b}=$ capacidade máxima de adsorção de $\mathrm{P}$.

$K=$ constante de energia de retenção de $P$.

$1 / b=$ inclinação da reta ou isoterma (coeficiente angular).

Porções de $5 \mathrm{~g}$ de terra foram transferidas para frascos de Erlenmeyer de $250 \mathrm{ml}$ aos quais se acrescentaram, em duplicata, $10 \mathrm{ml}$ de 
solução de $\mathrm{Ca} \mathrm{Cl}_{2} \quad 0,05 \mathrm{M}$ e quantidades convenientes de solução de $\mathrm{KH}_{2} \mathrm{PO}_{4}$ e água destilada de modo a se obterem volumes finais de $50 \mathrm{ml}$. As quantidades de $\mathrm{KH}_{2} \mathrm{PO}_{4}$ empregadas foram as necessárias para dar a volumes de $50 \mathrm{ml}$ de solução as seguintes concentrações de $\mathrm{P}$ (Tabela 2).

Tabela 2 - Concentração de $\mathrm{P}$ em $50 \mathrm{ml}$ de solução.

\begin{tabular}{|c|c|c|c|}
\hline \multirow{2}{*}{ Tratamento } & \multicolumn{3}{|c|}{ Concentração de $\mathrm{P}, \mu \mathrm{g} / \mathrm{ml}$} \\
\hline & Horizonte $\mathrm{A}_{1}$ & Horizonte $\mathrm{A}_{3}$ & Horizonte $\mathrm{B}_{22}$ \\
\hline 0 & 0 & 0 & 0 \\
\hline 1 & 10 & 20 & 20 \\
\hline 2 & 20 & 30 & 40 \\
\hline 3 & 30 & 40 & 60 \\
\hline 4 & 40 & 50 & 80 \\
\hline 5 & 50 & 60 & 100 \\
\hline 6 & 60 & 80 & 120 \\
\hline 7 & 80 & - & 140 \\
\hline
\end{tabular}

Os erlenmeyers foram colocados em agitador sendo agitados durante 17 horas. As suspensões foram, a seguir, centrifugadas a 2.500/3.000 rpm durante 15 minutos, determinando-se o teor de $\mathrm{P}$ no sobrenadante pelo método do reativo sulfo-bismuto-molíbdico, proposto por CATANI \& JACINTHO (1974).

\section{RESULTADOS E DISCUSSÃO}

Os resultados obtidos se encontram na Tabela 3 .

Tabela 3 - Concentração de $\mathbf{P}$ na solução de equilíbrio.

\begin{tabular}{cccc}
\hline \multirow{2}{*}{ Tratamento } & \multicolumn{2}{c}{ Concentração de $\mathrm{P}$ na solução de equilíbrio, $\mu \mathrm{g} / \mathrm{ml},(\mathrm{C})$} \\
\cline { 2 - 4 } & Horizonte $\mathrm{A}_{1}$ & Horizonte $\mathrm{A}_{\mathbf{3}}$ & Horizonte $\mathrm{B}_{22}$ \\
\hline \hline 1 & 0,000 & 0,000 & 0,000 \\
2 & 0,030 & 0,030 & 0,005 \\
3 & 0,080 & 0,056 & 0,018 \\
4 & 0,133 & 0,130 & 0,045 \\
5 & 0,330 & 0,222 & 0,195 \\
6 & 0,483 & 0,480 & 0,552 \\
8 & 0,940 & 1,400 & 1,245 \\
\hline
\end{tabular}


Com os dados da Tabela 3 foram calculadas as quantidades sorvidas de $\mathrm{P}$, em $\mu \mathrm{g} / \mathrm{g}$ de terra $(\mathrm{x} / \mathrm{M})$ e as relações $\frac{\mathrm{C}}{\mathrm{x} / \mathrm{M}}$.

Determinadas as equações de regressão linear $\frac{\mathrm{C}}{\mathrm{x} / \mathrm{M}} \cdot 10^{*} \mathrm{x} \mathrm{C}$ foram calculados os valores de adsorção máxima (b) e a constante de seletividade K. Os resultados estão na Tabela 4.

Tabela 4 - Valores de adsorção máxima (b), energia de retenção do $P$ (K), equações lineares e Langmuir e coeficientes de correlação para os 3 horizontes.

\begin{tabular}{|c|c|c|c|c|}
\hline Horizonte & $\begin{array}{c}\mathrm{b} \\
\mu \mathrm{g} \mathrm{P} \text { ads. por } \\
\mathrm{g} \text { de terra }\end{array}$ & $\begin{array}{c}\mathrm{K} \\
\mathrm{ml} / \mu \mathrm{g} \mathrm{P} \text { na } \\
\text { solução equilíbrio }\end{array}$ & Equação de regressão & $\begin{array}{l}\text { Coeficiente } \\
\text { de } \\
\text { Cerrelação }\end{array}$ \\
\hline$A_{1}$ & 642,78 & 5,97 & $\frac{\mathrm{C}}{\mathrm{x} / \mathrm{M}} \cdot 10^{4}=\underset{15,5572 \mathrm{C}}{2,6041+}$ & $0,995 * *$ \\
\hline$A_{3}$ & 763,99 & 9,78 & $\frac{\mathrm{C}}{\mathrm{x} / \mathrm{M}} \cdot 10^{4}=\frac{1,3383+}{13,0891 \mathrm{C}}$ & $0,995 *_{2}$ \\
\hline $\mathrm{B}_{22}$ & 1038,52 & 30,23 & $\underset{\mathrm{x} / \mathrm{M}}{\mathrm{C}} \cdot 10^{4}=\underset{9,6290 \mathrm{C}}{0,3185}+$ & $0,998 * *$ \\
\hline
\end{tabular}

** significativo a $1 \%$ de probabilidade.

Observa-se uma grande diferença tanto nos valores de adsorção máxima como nos da constante de seletividade, que para os horizontes $A_{3}$ e $B_{22}$ se apresentaram muito altos.

As isotermas de adsorção são mostradas na Fig. 1. A Fig. 2 apresenta os resultados da aplicação da equação de Langmuir aos dados de adsorção de $P$. Os pontos usados para traçar as isotermas de Langmuir correspondem à região de adsorção II proposta por MULJADI et al (1966) pois as concentrações da região I são muito baixas para serem avaliadas com precisão.

A Fig. 2 mostra uma boa adequação dos dados de adsorção de $P$ à equação de Langmuir pois foram obtidas relações lineares para os três horizontes com coeficientes de correlação altamente significativos (Tabela 4).

Para tentar explicar as diferenças encontradas nos três horizontes quanto aos parâmetros $\mathrm{b}$ e $\mathrm{K}$ da equação de Langmuir foram efetuadas correlações e ajustadas equações de regressão linear entre os referidos 


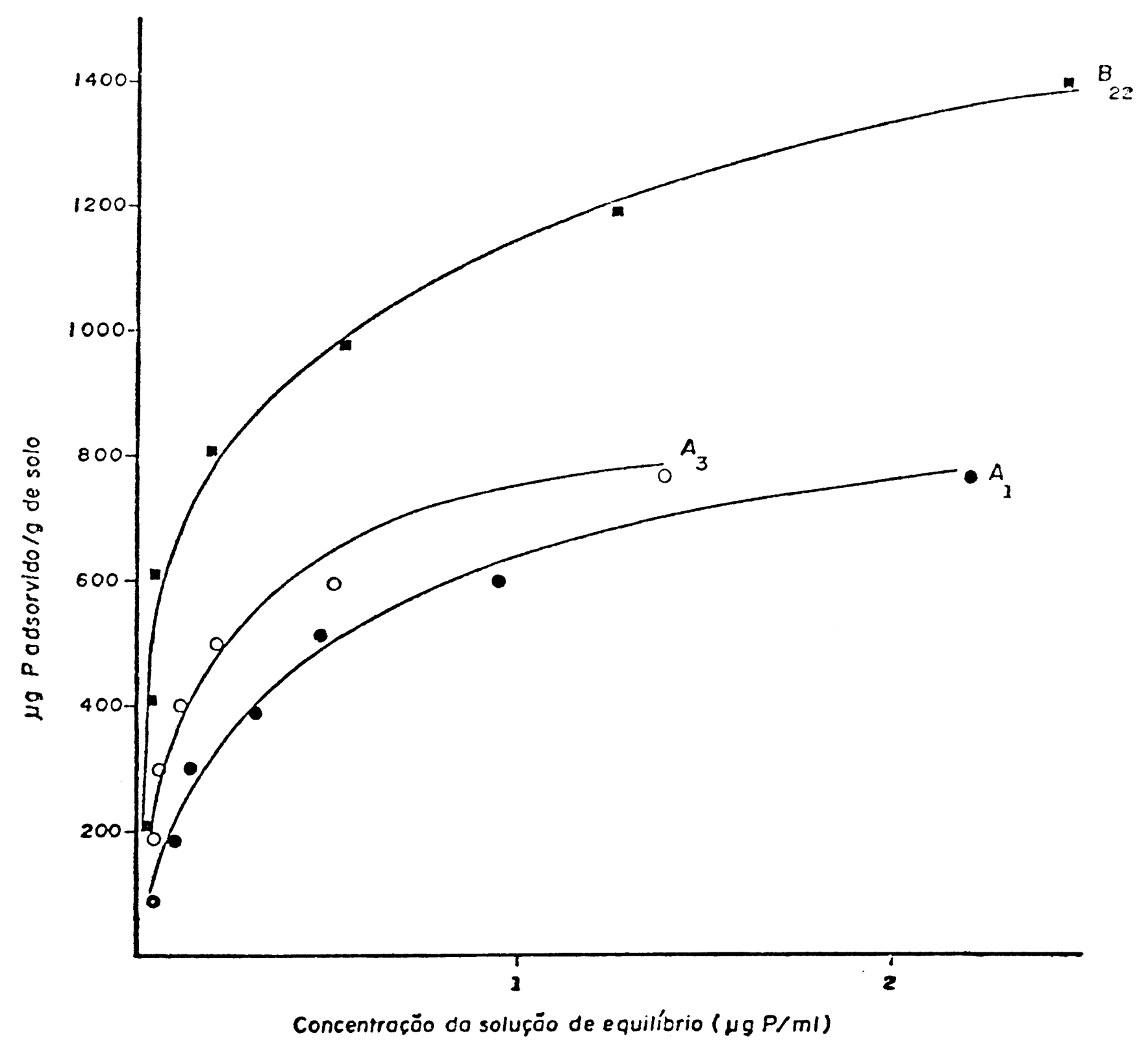

FIGURA 1 - Isotermas de adsorção para os horizontes $A_{1}, A_{2}$ e $B_{92}$ do Latossolo 


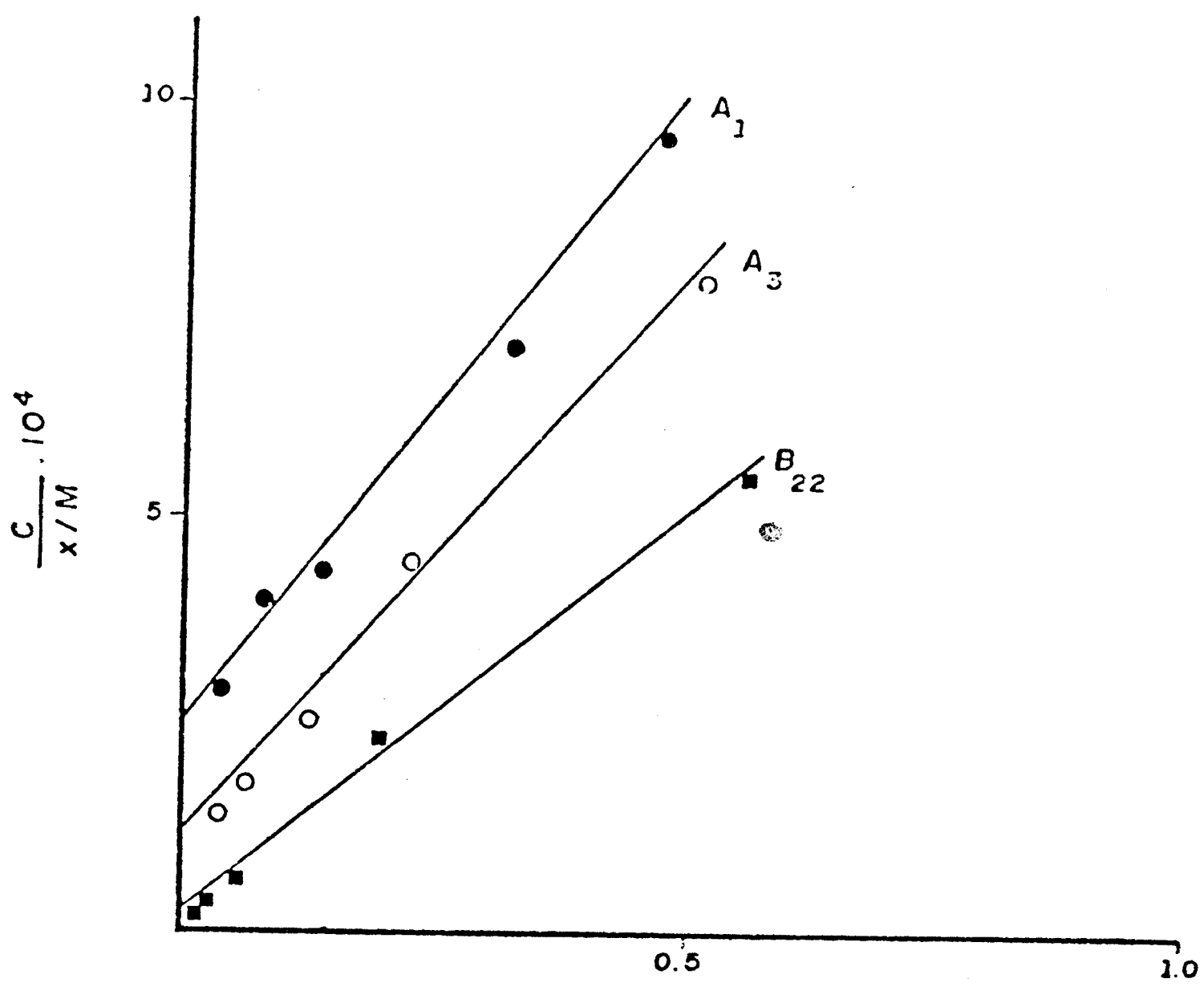

Concentroçăo do soluçäo de equiliorio $(\mu \mathrm{g} P / \mathrm{m} /)$

FIGURA 2 - Isotermas de adsorção de Langmuir para os horizontes $\mathrm{A}_{1}, \mathrm{~A}_{2}$ e $\mathrm{B}_{92}$ do Latossolo. 
parâmetros e algumas características físicas e químicas dos horizontes. Os resultados encontrados estão na Tabela 5 .

Tabela 5 - Relações entre as constantes $\mathrm{b}$ e $\mathrm{K}$ de Langmuir e algumas características dos horizontes estudados.

\begin{tabular}{|c|c|c|c|}
\hline $\begin{array}{l}\text { Correlação } \\
\text { estudada }\end{array}$ & & Equação de regressão & $\begin{array}{l}\text { Coeficiente de } \\
\text { correlação }\end{array}$ \\
\hline $\mathrm{b} \times \mathrm{x}$ argila $\%$ & $\mathrm{y}$ & $=-3288,881+48,122 \times$ & 0,557 \\
\hline $\mathrm{b} \times \mathrm{St} *$ & $\mathrm{y}$ & $560,353+$ & 0,223 \\
\hline $\mathrm{b} \times \mathrm{pH}$ & $\mathrm{y}$ & $=-3933,783+989,350 \mathrm{x}$ & 0,975 \\
\hline $\mathrm{b} \times \mathrm{C} \%$ & $\mathrm{y}$ & $=1121.531-150,213 \mathrm{x}$ & $-0,946$ \\
\hline b $\times \mathrm{Fe}_{2} \mathrm{O}_{3}$ livre $\%$ & $\mathrm{y}$ & $=-1219,050+133,241 \mathrm{x}$ & 0,589 \\
\hline $\mathrm{b} \times \mathrm{CTC}$ & $\mathrm{y}$ & $=1268,688-40,987 \times$ & $-0,953$ \\
\hline b $x \mathrm{Ki}$ & $\mathrm{y}$ & $1488,087-548,633 x$ & $-0,810$ \\
\hline b $\times \mathrm{Kr}$ & $\mathrm{y}$ & $1469,690-821,666 x$ & $-0,787$ \\
\hline $\mathrm{K} \times$ argila $\%$ & $\mathrm{y}$ & $183,893+2,336 x$ & 0,420 \\
\hline $\mathrm{K} \times \mathrm{St} *$ & $\mathrm{y}$ & $10,163+0.081 \mathrm{x}$ & 0,070 \\
\hline $\mathrm{K} \times \mathrm{pH}$ & $\mathrm{y}$ & $275,793+60,650 x$ & 0,929 \\
\hline $\mathrm{K} \times \mathrm{C} \%$ & $\mathrm{y}$ & $33,749-9,031 \times$ & $-0,884$ \\
\hline $\mathrm{K} \times \mathrm{Fe}_{2} \mathrm{O}_{3}$ livre $\%$ & $\mathrm{y}$ & $85,937+$ & 0,676 \\
\hline $\mathrm{K} \times \mathrm{CTC}$ & $\mathrm{y}$ & $42,713-2,475 x$ & $-0,895$ \\
\hline $\mathrm{K} \times \mathrm{Ki}$ & $\mathbf{y}$ & $53,197-30,873 x$ & $-0,108$ \\
\hline $\mathrm{K} \times \mathrm{Kr}$ & $\mathrm{y}$ & $51,751+45,721 \mathrm{x}$ & $-0,679$ \\
\hline
\end{tabular}

* Superfície específica total.

Não obstante ter sido apenas três o número de pares de dados utilizados para os cálculos, não permitindo, de certa forma, o teste de significância estatística dos coeficientes de correlação encontrados, os mesmos mostram as tendências da influência de cada característica na adsorção do fósforo.

Os resultados encontrados quando se correlacionou adsorção máxima (b) $\mathrm{x}$ teor de carbono não concordam com os encontrados por LEAL \& VELOSO (1973), LOURENÇO (1973) e BITTENCOURT \& ZAMBELLO (1973) os quais encontraram correlação significativa e positiva entre adsorção máxima e carbono.

Embora sendo um Latossolo, o solo utilizado apresenta um teor de argila que aumenta com a profundidade, o que, de certa maneira, 
influenciou na maior adsorção de fósforo pelas amostras dos horizontes sub-superficiais, resultados que estão de acordo com os encontrados por LEAL \& VELOSO (1973) e SÁ JR. (1969), mas que não concordam com os obtidos por FASSBENDER (1969).

Os coeficientes de correlação encontrados para as demais variáveis independentes apresentam valores relativos concordantes com os sugeridos por BRAGA (1973), com exceção do $\mathrm{pH}$, como pode ser visto na Tabela 6.

Tabela 6 - Valores relativos dos coeficientes de correlação entre adsorção máxima e características químicas do Latossolo utilizado e os valores respectivos sugeridos por BRAGA (1973).

\begin{tabular}{ccc}
\hline & \multicolumn{2}{c}{ Valores relativos dos coeficientes $\mathrm{r}$} \\
\cline { 2 - 3 } & BRAGA (1973) & encontrado \\
$\mathrm{nH}$ & - & + \\
$\mathrm{Fe}_{2} \mathrm{O}_{3}$ livre & + & + \\
$\mathrm{CTC}$ & - & - \\
$\mathrm{Ki}$ & - & - \\
$\mathrm{Kr}$ & - & - \\
\hline
\end{tabular}

\section{CONCLUSÕES}

As principais conclusões que se podem tirar do trabalho são as seguintes:

a - As isotermas de adsorção mostraram duas regiões distintas; aquela em que o fosfato é fortemente retido foi convenientemente descrita pela equação de Langmuir.

b - Em virtude da diversidade das características físicas e químicas dos horizontes houve grande variação nos valores de adsorção máxima (b) e da constante de seletividade (K). 
SUMMARY

\section{PHOSPHORUS ADSORPTION BY A LATOSSOL FROM THE STATE OF MINAS GERAIS, BRASIL}

It was studied the adsorption of $P$ by samples of the horizons $A_{1}(0-22 \mathrm{~cm}), A_{3}(22-56 \mathrm{~cm})$ and $\mathrm{B}_{2^{2}}(155-200 \mathrm{~cm})$ of a Latossol from the State of Minas Gerais, Brasil, by mean of the isotherm of Langmuir.

The maximum adsorption values (b) and selectivity constant $(\mathrm{K})$ calculated from the linear pattern of the Langmuir equation were correlated with some physical and chemical characteristics of the horizons.

The main conclusion were as follows:

a) The adsorption isotherm showed two definite regions: the one regarding to the high $P$ fixation is in accordance with the Langmuir equation.

b) It was observed big differences in respect to the values of maximum adsorption (b) as well as to the selectivity constant $(K)$.

\section{LITERATURA CITADA}

BITTENCOURT, V.C. \& E.ZAMBELlO JR., 1973 - Comportamento do fósforo em solos de regiões tropicais. I. Isotermas de adsorção. Bol. Cient. CENA, n. ${ }^{\circ}$ 012, 23 p., Piracicaba.

BRAGA, J.M., 1973 - Fixação de fósforo. Seminário, 20 p., mimeografadas.

CATANI, R.A. \& A.O. JACINTHO, 1974 - Análise química para avaliar a fertilidade do solo. Bol. Tec. Cient., Esc. Sup. Agric. "Luiz de Queiroz", n. 37,54 p., Piracicaba.

FASSBENDER, H.W., 1969 - Estudio del fósforo em suelos de America Central. IV. Capacidade de fijación de fósforo y su relación com características edáficas. Turrialba, $19: 497-505$.

GROHMANN, F., 1972 - Superfície específica do solo de unidades de mapeamento do Estado de São Paulo. I - Estudo de perfis com horizonte B textural e horizonte B latossólico. Bragantiá, 31:145-165.

GROHMANN, F. \& B. VAN RAIJ, 1974 - Influência dos métodos de agitação na dispersão da argila do solo. Anais do XIV Congr. Bras. de Ciência do Solo: 123-132. Santa Maria, R.S.

HEILMAN, M.D., K.L. CARTER \& C.L. GONZALEZ, 1965 - The etylene glycol monethyl eter technique for determining soils surface area. Soil Sci. $100: 409-413$.

JACKSON, L.T., 1965 - Soil Chemical Analysis: Advanced Cource, 991 p. Dept. of Soil Science, Madison. 
LEAL, J.R. \& A.C.X. VELOSO, 1973 - Adsorção de fosfato em Latossolos sob vegetação de cerrado. Pesq. Agrop. Bras. Ser. Agron. 8:81-88. Rio de Janeiro.

LOURENÇO, S., 1973 - Adsorção e desorção do fósforo em solos do Estado do Paraná. Tese de Doutoramento, 69 p., ESALQ, Piracicaba.

MULJADI, D., A.M. POSNER \& J.P. QUIRK, 1966 - The mechanism of phosphate adsorption by kaolinite, gibsite and psudbohemite. Part I. The isotherms and the effect of $\mathrm{pH}$ on adsorption. J. Soil Sci. $17: 212-229$.

OLSEN, E.R. \& F.S. WATANABE, 1957 - A method to determine phosphorus adsorption maximum of soils as measured by Langmuir isotherm. Proc. Soil Soc. Am., 21: 144-149, Ann. Arbor.

SÁ JR., J.P.M., I.F. GOMES \& A.L. VASCONCELLOS, 1968 - Retenção de fósforo em solos da zona da Mata de Pernambuco. Pesq. Agrop. Bras. Ser. Agron. 3: 183-188. Rio de Janeiro.

VETTORI, L., 1969 - Métodos de análise do solo. Bol. Tec. Equipe Pedol. Fertil. Solo, n..$^{\circ}$, 24 p., Rio de Janeiro. 
